\title{
Elastomeric Materials
}

Because rubber plants occur naturally in only a few out-of-the way places around the world-such as Malaya, South America, and Indonesia-attempts to create synthetic materials with the useful properties of rubber began a century and a half ago. The many investigations eventually resulted in a variety of new elastomeric materials and also laid the basic foundations for polymer chemistry. Chemists finally succeeded in synthesizing the actual rubber molecule in the late 1950 s, but by that time other elastomers had found broad applications in worldwide technology and industry.

The synthesis of rubber from other raw materials could not begin until the basic chemistry of the substance was better understood. In 1826 in England, Michael Faraday made one of the first chemical analyses of natural rubber, interpreting its molecular formula as $\mathrm{C}_{5} \mathrm{H}_{8}$. With this knowledge, many other hopeful researchers attempted to build synthetic rubber molecules that could be produced easily and inexpensively. In 1838 in Germany, F.C. Himly was able to obtain a volatile distillate from natural rubber, and in 1860 in England, chemist C.G. Williams went further and distilled natural rubber into three parts, oil, tar, and "spirit" (the volatile fraction). He named the "spirit" isoprene.

The next step was to see if isoprene could be synthesized from readily available raw materials and then converted back into rubber. In France, Georges Bouchardat used hydrogen chloride gas in 1875 in a prolonged distillation of isoprene, which yielded a rubberlike substance. This was one of the first synthetic elastomers ever created.

In 1882 in England, W.A. Tilden produced isoprene through the destructive distillation of turpentine, rather than any raw material that came from natural rubber. He reported that, after storage or through the action of chemical agents, his specimens of isoprene changed into a material that resembled rubber.

The search for substitutes for natural rubber did not make major progress until researchers stopped trying to reproduce the exact chemical composition of rubber, and instead concentrated on synthesizing materials with similar properties.

Such rubbery materials, with chemical structures very different from rubber, required a more general designation, though: "Elastomer," a contraction of elastic and polymer, was defined as a substance that can be stretched at room temperature to at least twice its original length and, after being stretched, rapidly returns to approximately its original length when the stress is removed.

For a material to exhibit elastomeric properties, it must have long threadlike molecules, flexibility in the molecular chain to allow stretching and coiling, and some form of chemical or mechanical bonds between the molecules. Petroleum has been the most important raw material for creating synthetic elastomers; some synthetic rubbers also use potatoes and grains (and the alcohol produced by them), coke, limestone, salt, and sulfur.

Germany and Russia, who had no ready access to plantation rubber, began to work in earnest on the problem at the turn of the century. In 1901 I. Kondakov produced a rubberlike substance by heating dimethylbutadiene with potash; and in 1910 S.V. Lebedev polymerized butadiene (which he had obtained from ethyl alcohol made out of potatoes) into an acceptable elastic material.

In the United States, Kyrides and Earle at the Hood Rubber Company synthesized dimethylbutadiene and polymerized it into synthetic rubber; David Spence of the Diamond Rubber Company synthesized isoprene from turpentine, then prepared synthetic rubber in a pilot plant.

One "failure" in the
search for synthetic
rubber was Silly Putty.

Hoffman and Harries in Germany, and Mathews and Strange in England, independently studied the polymerization of butadiene, isoprene, and dimethylbutadiene. They discovered how to prepare synthetic elastomers from these base materials by using metallic sodium as a general polymerizing agent. During World War I, while Germany was under a naval blockade, 2,300 tons of "methyl rubber" were manufactured by this process-

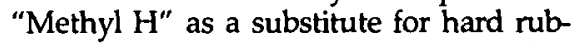
bers and "Methyl W" for soft rubber applications. Shortages forced the use of dimethylbutadiene as the base material, rather than the more satisfactory butadiene or isoprene. Methyl rubber proved to be a poor substitute for natural rubber, and it was abandoned immediately after the war.
In the late 1920 s in Germany, Staudinger laid the foundations of basic polymer chemistry, and a boom in synthetic elastomers occurred. In the United States, with ample supplies of natural rubber, interest in developing synthetics waned. However, some companies showed increasing interest in developing elastomeric materials with particular physical and chemical properties for special applications that needed, for instance, greater chemical resistance or greater solvency than natural rubber.

The first such special elastomer, polysulfide rubber, better known by the trade name of Thiokol ${ }^{\oplus}$, was marketed in 1930 by the Thiokol Chemical Corporation. J.C. Patrick discovered Thiokol in 1920 while trying to prepare a new antifreeze from ethylene dichloride and sodium polysulfide; instead, he found a new rubbery substance that was not marketed until a decade later. While Thiokol has a much lower tensile strength than natural rubber, it also shows excellent resistance to organic solvents such as gasoline, and it resists weathering and gas permeation over a wide range of temperatures.

Another synthetic elastomer, polychloroprene, better known by its trade name of Neoprene ${ }^{\circledast}$, was first marketed in 1931 by E.I. du Pont de Nemours \& Co. It was discovered by J.A. Nieuwland, a professor of chemistry at the University of Notre Dame, assisted by W.H. Carothers (creator of nylon) and Elmer K. Bolton. Scientists in the Soviet Union also manufactured polychloroprene, which they called "Sovprene."

Neoprene is manufactured from acetylene, made from coal, limestone, and water. It has a high tensile strength and resilience, good tear resistance, and good resistance to oil, oxygen, and ozone. Neoprene is used to manufacture gloves, tubes, hose, belts, gaskets, and some extruded mechanical parts.

Researchers in Germany were successful with their Buna rubbers, introduced commercially in 1935, made from sodium polymerization of butadiene and styrene. (The name "Buna" comes from butadiene and the chemical symbol for sodium, $\mathrm{Na}$.) Butadiene-styrene rubber, known as Buna$S$, is the most widely used synthetic elastomer and has physical characteristics very similar to natural rubber. It ages well and has good abrasion and crack resistance, though it has low strength (unless reinforced with fillers such as carbon black), low resilience, and low tear strength.

Another buna rubber used in Germany, Buna- $\mathrm{N}$ or nitrile, was introduced to the United States in 1937. Highly resistant to oil, nitrile is used in seals and gaskets and 
other places where the rubber comes in contact with hot oils, for example the rollers in printing presses and hoses used for oil products.

The United States began manufacturing butadiene-styrene synthetic rubber when, in World War II supplies of natural rubber were cut off from the Far East. The U.S. synthetic elastomer industry had been insignificant, but at the time of the blockade only about 1 million tons-or one year's supply-of natural rubber existed in the country. The synthetic rubber industry had to begin producing almost overnight, achieving 800,000 tons per year. While improvements in the process were made during wartime, the U.S. government retained the same butadiene-styrene manufacturing methods until the end of the war to avoid confusion.

Butyl rubber (polyisobutylene) was discovered in 1937 by R.M. Thomas and W.J. Sparks, and introduced to the market in 1943 by the Standard Oil Company. Butyl rubber has become popular because of its resistance to vegetable oils, oxygen, and ozone. It has a low gas permeability, and besides good resistance to acids and other chemicals harmful to natural rubber, butyl rubber is light in color, nontoxic, elastic, odorless, and has good electrical insulation properties. Disadvantages include its stiffness at low temperatures and sensitivity to contamination during processing (even small amounts of other rubbers or plasticizers will interfere with proper vulcanization). Butyl rubber is used for inner tubes, protective clothing, and window strips in automobiles.

Not until 1956 was natural rubber first synthesized in the laboratory. H.J. Teas and R.S. Banduski announced that they had formed small samples of natural rubber molecules out of acetate by using enzymes obtained by centrifuging latex from the rubber plant.

By this time, though, the development of the petrochemical industry had brought about so many different synthetic elastomers that natural rubber was no longer so important to everyday life and for growing industries. By the way, one "failure" in the search for synthetic rubber was the development of the elastomer known as Silly Putty ${ }^{\oplus}$, a popular children's toy for four decades now.

The demand for synthetic elastomeric materials has increased each year, and new elastomers continue to be developed with physical characteristics appropriate and desirable for every application formerly dominated by natural rubber. By the mid1970 s, worldwide consumption of synthetic rubbers was nearly twice that of the natural product. The actual tonnage of natural rubber continued to increase through the 1980s, during the worldwide growth of industry, but synthetic rubbers have consistently claimed a larger percentage even though virtually all of these substitutes were completely unknown only a century before.

KEVIN J. ANDERSON

Editor's Note: See "Rubber Stretches Over Two Anniversaries" (July 1988, p. 47) and "Large-Scale Dilatant Dynamics of Silly Putty" (February 1990, p. 69) in the MRS BULLETIN.

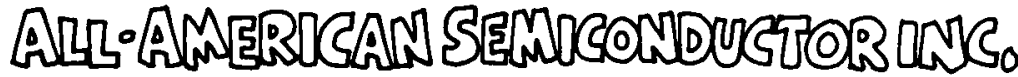

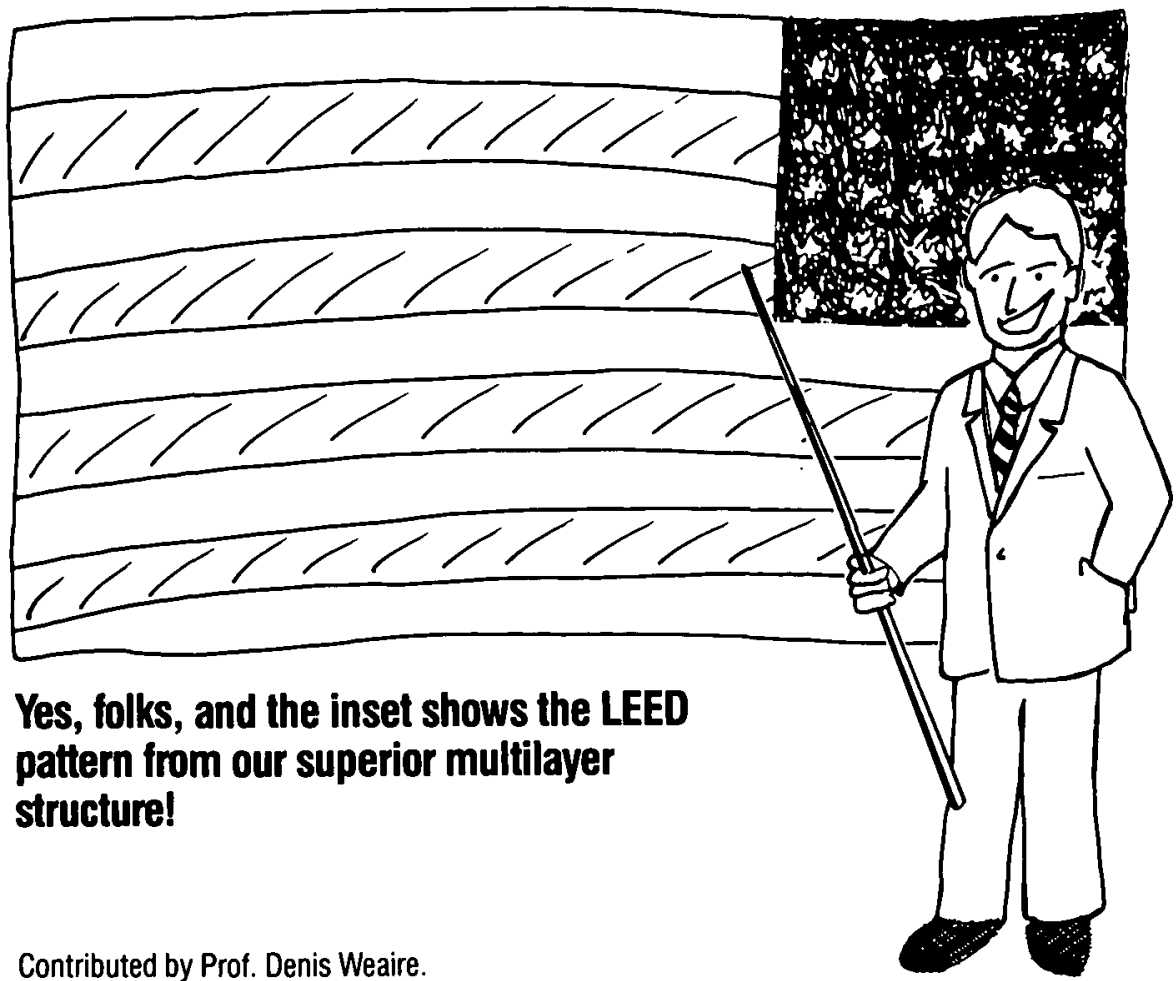

\section{In the next issue...}

Guest Editor Alan R. Krauss of the Materials Sciences and Chemistry Divisions at Argonne National Laboratory tums the focus on "New Materials and New Challenges in Vacuum Technology" in a series of six articles:

"Developments and Applications of All-Aluminum Alloy Vacuum Systems" by $\mathrm{H}$. Ishimaru

"Surface Preparation and Coating of Aluminum Alloys for Improved Vacuum Properties' by K. Moriyama

"Vacuum Systems for Synchrotron Light Sources" by J.N. Schuchman

"Review of Wall Conditioning and Wall Materials for Fusion Research Devices' by R.A. Langley

"Helium Pumping Strategies for D-T Fusion Devices" by A. Krauss, J.N. Brooks, 0. Auciello, and W. Shmayda

Non-Evaporable Getters: Properties and Applications" by C. Boffito and F. Mazza 
UHV ECR Source

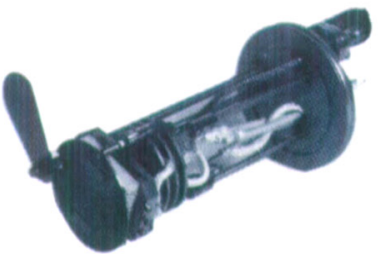

1000 Degree C Heater

The MICRO1000 range of heaters operate up to 1000 degrees $C$. even in an oxygen environment. Available in a number of configurations to fit any vacuum system. Comes with a range of optional accessories including shutter, cooling shroud and DC and RF biasing. For more information call Microscience, 41 Accord Park Dr., Norwell, MA 02061. Tel. (617) 871-0308.

\section{Small Batch Sputter Production Systems}

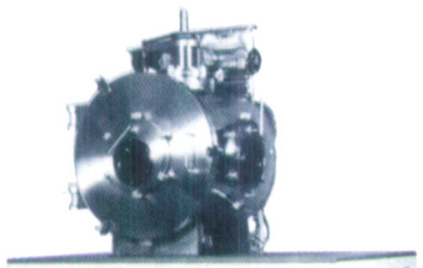

The Microscience MicroProd sputter system is suitable for prototype and batch production work with small substrates. Features a 24 inch dia. barrel chamber, loading door, carousel sample holder, and a variety of ports and feed throughs for magnetron sputter guns and ion sources. The MicroProd is a CAD design that can be easily adapted for a wide range of uses. Contact Microscience, 41 Accord Park Dr. Norwell, MA 02061. Tel. (617) 871-0308.

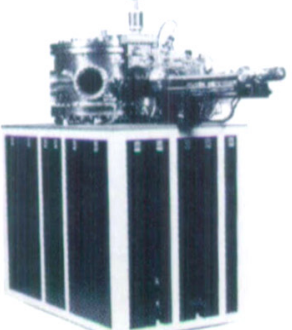

\section{Researcher i01}

Microscience introduces the researcher 101. An integrated UHV thin film system for advanced R\&D applications. Originally designed for research on HTC films, the 101 incorporates many specialized features including transportable 1000 degree heater substrate carrier, stepper motor controlied. substrate positioning system and optional surface science module. The 101 is offered in HV and UHV configurations and can be fitted with a wide range of tools including planar magnetrons, ion sources and an ECR plasma stream source. From Microscience, 41 Accord Park Dr., Norwell, MA 02061. Tel. (617) 871-0308.
The Microscience 904 ECR source is designed for retrofitting to UHV chambers. The source comes complete with microwave circuitry, magnets and power supplies.

Details from Microscience,

41 Accord Park Dr., Norwell, MA 02061. Tel. (617) 871-0308.

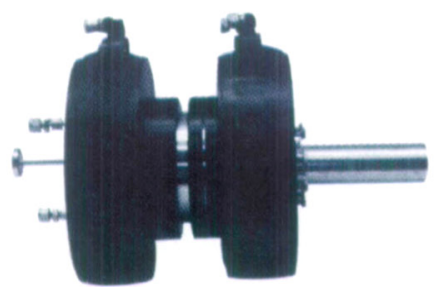

MBE Sputter System

The Microscience 401 sputtering system is designed for interfacing to MBE systems. Features include a transfer system, load lock, rotating heated biasable sample holder magnetron cluster and an LN cryoshroud. This is a CAD based design that can be adapted for most MBE systems. Contact Microscience, 41 Accord Park Dr., Norwell, MA 02061. Tel. (617) 871-0308.

The ECR $908 \mathrm{G}$ is a high efficien ECR plasma stream source fitted with extraction grids for energies in the range $50-5000 \mathrm{eV}$. The $908 \mathrm{G}$ produces beam densities up to $100 \mathrm{~mA} / \mathrm{cms}$. Applications include ion beam enhanced deposition, ion etching and RIBE. Contact Microscience, 41 Accord Park Dr., Norwell, MA 02061. Tel. (617) 871-0308.

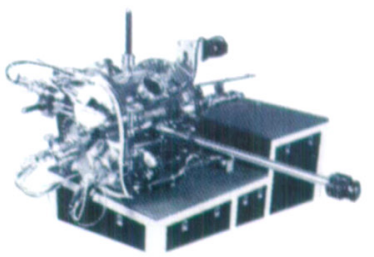

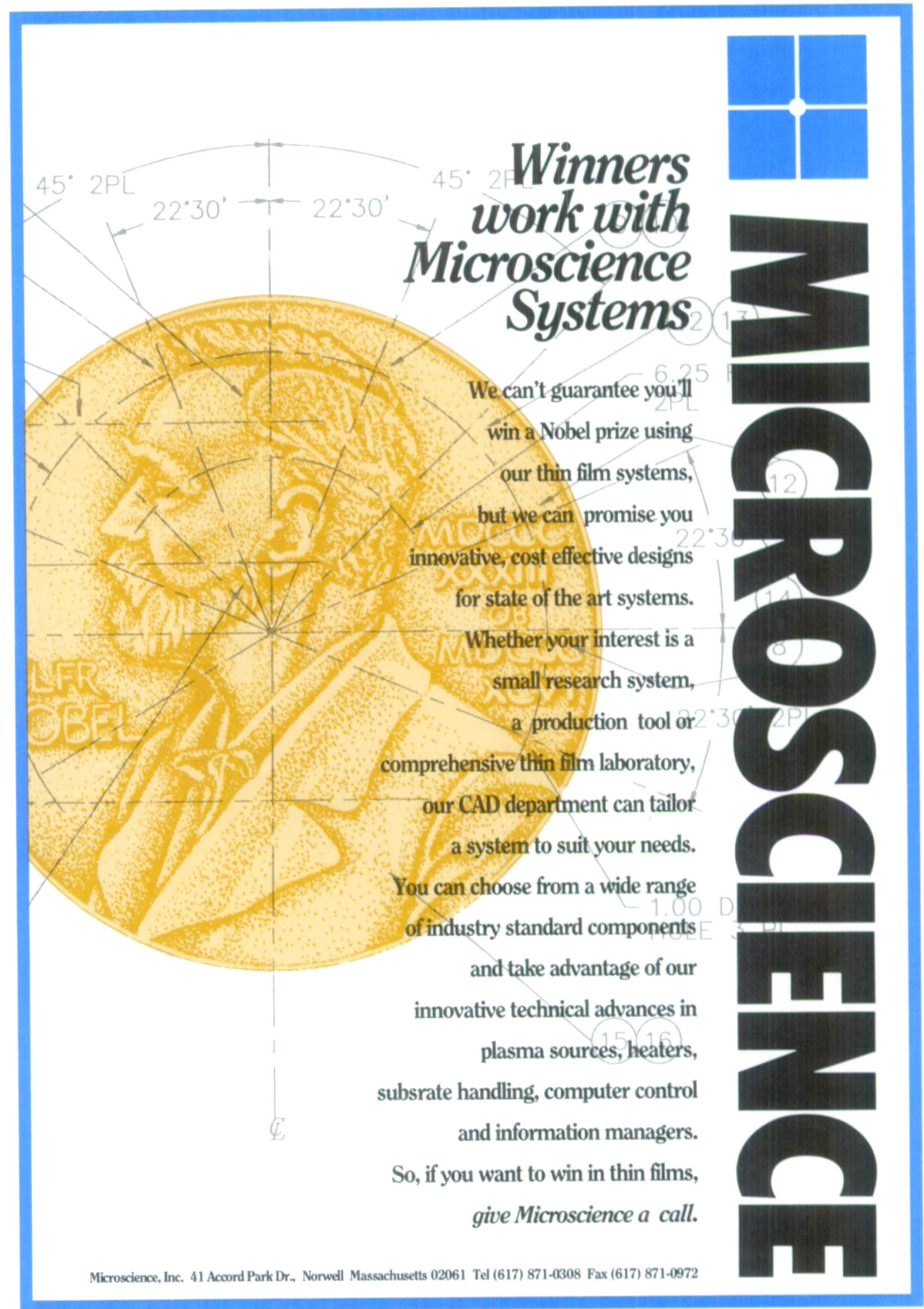




\section{Springer for Materials Science}

Theoretical and Applied Books on Superconductivity from Springer-Verlag

Now available-

J.W. Lynn, University of Maryland, College Park, (ed.)

High Temperature Superconductivity

Through contributions by key researchers at the University of Maryland and throughout the United States, this text provides a comprehensive overview of the theory of superconductivity and the new high-Tc oxides. Contributors include J.W. Lynn, D. Belitz, R.A. Ferrell, A. Santoro, C.-p.S. Wang, R.N. Shelton, J.E. Crow, N.-P. Ong, P.B. Allen, and F.D. Bedard.

$1990 / 403$ pp., 125 illus./Hardcover $\mathbf{\$ 3 9 . 9 5}$

ISBN 0-387-96770-2

Graduate Texts in Contemporary Physics

J.G. Bednorz and K.A. Muller, both, IBM Research

Laboratory, Ruschlikon, Switzerland (eds.)

Earlier and Recent Aspects of

Superconductivity

International School, Erice (Sicily), Italy

July 4-16, 1989

Intended for students and researchers entering the field, these contributions incorporate the latest developments in high-Tc cuprites into the 80 year history of classical superconductivity. The book provides a coherent overview of fundamental properties, categorization, structure and electronic properties, properties related to coherence length, and theoretical models. 1990/approx. 464 pp., 280 illus., 17 tables/Hardcover/\$39.95 ISBN 0-387-52156-9

Springer Series in Solid-State Sciences, Vol. 90

In preparation-

B. Raveau, C. Michel, M. Hervieu, and D. Groult

Crystal Chemistry of High Tc

Superconducting Copper Oxides

1990/Hardcover ISBN 0-387-51545-3

Springer Series in Materials Science, Vol. 15

Due: September 1990

M.A. Savchenko and A.V. Stefanovich, both, Moscow, USSR Fluctuational Superconductivity of Magnetic Systems

Addressing physicists and chemists investigating and synthesizing new high-temperature superconducting materials, this book proposes methods of increasing the critical temperature of high-temperature superconductors.

1990/approx. 257 pp., 64 illus./Softcover $/ \$ 49.50$

ISBN 0-387-50561-X

Research Reports in Physics

H. Fukuyama, U. of Tokyo, Japan, S. Maekawa, Nagoya U., Japan, and A.P. Malozemoff, IBM, Yorktown Heights, NY (eds.)

Strong Correlation and Superconductivity

Leading physicists report on experimental and theoretical investigations into high-Tc superconductivity, with emphasis on the basic physics.

1989/396 pp., 198 illus./Hardcover/\$59.00

ISBN 0-387-51320-5

Springer Series in Solid-State Sciences, Vol. 89
T. Ishiguro, Kyoto U., Japan, and K. Yamaji, Electrochemical Lab., Ibaraki, Japan

\section{Organic Superconductors}

Provides an introduction to organic conductors and superconductors, and reviews the current status of the field. Discusses the structures and electronic properties of superconductors and contains illustrations of typical molecules.

1990/288 pp., 189 illus./Hardcover/\$59.50

ISBN 0-387-51321-3

Springer Series in Solid-State Sciences, Vol. 88

J.H. Hinken, Hans Kolbe \& Co., Bad Saldetfurth, FRG Superconductor Electronics

Fundamentals and Microwave Application

Presents the fundamentals of superconducting electronics in general and with specific applications to microwave engineering, e.g., SIS mixers and Josephson junctions. Practical aspects. including production and choice of materials, are covered, as well as cryogenic engineering and the impact of high-Tc superconductivity.

1989/158 pp., 94 illus./Hardcover $/ \$ 39.00$

ISBN 0-387-51114-8

Graduate Texts in Contemporary Physics

V. Kose, Physikalisch-Technische Bundesanstalt, Braunschweig, FRG (ed.)

Superconducting Quantum Electronics

Reviews recent accomplishments, presents new results, and discusses possible future developments of superconducting quantum electronics and high-Tc superconductivity. The three main parts of the book deal with fundamentals, sensitive detectors, and precision metrology.

1989/299 pp., 180 illus./Hardcover/\$49.50

ISBN 0-387-51176-8

H. Weinstock, Air Force Office of Scientific Research. Wash., D.C., and M. Nisenoff, Naval Research Lab., Wash.. D.C. (eds.) Superconducting Electronics

Provides an in-depth understanding of the fundamentals of superconducting electronics and the practical considerations for the fabrication of superconducting electronic structures.

$1989 / 441 \mathrm{pp} . /$ Hardcover $\$ \mathbf{\$ 7 . 0 0}$

ISBN 0-387-51521-6

NATO ASI Series F, Vol. 59

\footnotetext{
To Order: call TOLL FREE 1-800-SPRINGER (in NJ, 20 !$348-4033$ ) or send payment (including $\$ 2.50$ for shipping) to: Springer-Verlag New York, Inc., Attn: K. Quinn/Dept. 521, 175 Fifth Ave., New York, NY 10010. Residents of NY, NJ, $\mathrm{MA}$, and $\mathrm{CA}$, please add sales tax for books. Instructors may call 212-460-1577 for examination copy information. Outside North America: please order through your bookseller or from Springer for Science, POB 503, 1970 AM Ijmuiden, The Netherlands. Prices subject to change without notice.
} 